\title{
COVID-19 in Dental Disciplines, An Opportunity for the Application of Digital Resources
}

\author{
Letter to Editor
}

Volume 1 Issue 1- 2021

\author{
Author Details \\ Rolando Morales Espinosa* \\ Oral pathologist, Chile \\ *Corresponding author \\ Rolando Morales Espinosa, Oral pathologist, Servicio de Salud Viña del Mar-Quillota, Valparaíso, Chile \\ Article History \\ Received: March 26, 2021 Accepted: April 02, 2021 Published: April 05, 2021
}

Keywords: Dental disciplines; COVID-19; Telemedicine health programs

\section{Letter to Editor}

The pandemic associated to SARS-CoV-2 is a reality that affects all the planet to a greater or lesser extent and requires a social and political effort to prevent and foresee different propagation scenarios, where work and social activities have been affected by restriction measures, mainly seeking to avoid direct contact between people who are the potential contagion.

In this context, dentistry as a science and medical discipline has not been the exception. The dental clinic has been considered by different entities and various investigations as one of the potential sources of infection with the virus due to the release of aerosols and the direct contact of the medical staff with the patient [1], therefore that the decrease in dental activity means that the dentist must reinvent himself, both in the way of contacting and treating his patients as well as in the alternatives of specialization and training.

The crisis caused by COVID-19 is a challenge for academic entities and universities, forcing them to develop strategies and configure their curriculum from face-to-face to online platforms, both for dentists in undergraduate training as well as specialization and postgraduate programs. The use of video calls, group communication applications, videos and asynchronous activities have been strategies that have been refined as the pandemic progresses, and that although they were considered at some point as insufficient resources for the instruction of professionals, currently they have shown satisfactory results since the theoretical presentation of the necessary knowledge for professionals in training has been facilitated.

In addition, the last year we have seen how much of the current scientific events are transmitted online, allowing digital platforms to integrate with greater coverage to different professionals at a regional and global level, extending the academic and training network to a greater number of dentists [2].

It is these same digital platforms that have allowed specialists to communicate with other colleagues or with their patients for the resolution of cases, evaluation of examinations, application of pharmacological treatments and control of the disease, reducing the direct contact of the professional with the patient and the overexposure of the latter to hospitals or health centers, where it can potentially be infected, by implementing telematic or telemedicine health programs.

However, it should be noted that a large part of the digital path lacks the direct contact component, which affects the teacher-student and doctor-patient relationship, but also makes them actively responsible for their training or healing process. Likewise, it poses the challenge of improving the conditions and programs that allow not only the theoretical training of students, but also their professional practice, in what could be future digital simulators for the training of dentists, considering that the practice of rehabilitation and restoration techniques in dentistry are the basis of dental therapy, so the creation of digital tools that allow this function will be fundamental in the future, a post-pandemic future that will require not only maintaining a new normal, but also compatible with our profession and daily life.

\section{References}

1. Doriguetto PVT, Americano JP, Devito KL (2020) Challenges for the dental radiology clinic in times of the COVID-19 pandemic. Oral Radiol 36(4): 404-405.

2. Cral WC, Queluz DP (2021) Online scientific events: A post-pandemic? Int J Odontostomat 15(1): 1-11. 